\title{
On Regulation of Spectrum Sharing: An Analysis Supporting a Distributed Pilot Channel
}

\author{
Jens P. Elsner, Leonid Chaichenets, Hanns-Ulrich Dehner and Friedrich K. Jondral \\ Universität Karlsruhe (TH), Germany, \{elsner, chaichenets, dehner, fj\}@ int.uni-karlsruhe.de
}

\begin{abstract}
This paper argues in favor of introducing a standardized distributed pilot channel to improve spectrum sharing between heterogeneous technologies in unlicensed and possibly licensed bands with incumbent users. The main features of such a distributed pilot channel are sketched and a simple distributed algorithm for optimization of spectrum sharing is introduced. The pilot channel serves as a supplement to spectrum sensing and can improve spectrum sharing in unlicensed and licensed bands.
\end{abstract}

\section{INTRODUCTION}

Wireless devices operating in unlicensed bands, such as devices based on the IEEE 802.11 (WLAN) and the IEEE 802.15 (WPAN) families of standards, have become ubiquitous over the last decade. The main factor for the success of these devices is the world-wide availability of standardized "unlicensed" spectrum, namely the ISM bands. Unlicensed spectrum can be used by any user under a general authorization anywhere at any time. Only few technological aspects such as maximum transmission power, bandwidth or duty cycle are regulated.

In densely populated areas, however, the success of unlicensed spectrum has lead to a situation where interference between wireless networks is a limiting factor to performance: multiple networks and technologies are competing for spectrum. Within networks using a single technology, access to spectrum is governed by medium access control protocols. These protocols assure fair use of the available spectrum for every terminal in the technologically homogeneous network. Advanced protocols manage external interference to the network by choosing an appropriate less-crowded wireless channel for transmission and thereby maximize their own throughput and hence implicitly coordinate spectrum access. Between heterogeneous technologies, however, there is, up until now, no explicit coordination protocol for spectrum access. In licensed bands, which need performance guarantees for primary users, spectrum sharing has long been undermined by the fact that any uncoordinated interference can have an adverse effect on the incumbent users. Here, too, more efficient use of spectrum can be achieved by explicit coordination between primary and overlay users.

\section{Scope and Related Work}

Various research efforts are directed at the structural description and development of such coordination protocols, for licensed and unlicensed bands, most notably the efforts of the IEEE P1900.4 working group [1], [2].
Apart from the efforts of the IEEE, a theoretical analysis of the limits of spectrum sharing is given by Etkin, Grokop and Tse [3], [4]. They analyze spectrum sharing using a game theoretic approach. Their argument is based on games of complete information, which makes exchange of parameters according to a predefined protocol, much like a common pilot channel, necessary. Fundamental limits are derived based on information theoretic arguments, extending well beyond currently realizable wireless systems.

Zhao et al. [5] propose a distributed coordination scheme without a common pilot channel, but assume that all terminals implement the same protocol. Peng et al. [6] consider spectrum assignment as a centralized resource allocation problem with a binary interference metric.

In contrast to these works, the problem of spectrum sharing is treated here as a structurally simple optimization problem which takes the physical limitations of the high-frequency frontend into account. The approach is constructive to derive simple but efficient regulatory rules. Concepts for neighbor discovery traditionally found at the network layer of a wireless protocol are used to advertise spectrum usage and to generate an interference matrix or graph. This interference graph in turn allows to optimize spectrum usage in a decentralized manner. Spectrum is not explicitly coordinated - each network makes its own decisions. The possible performance of such a protocol is then evaluated in simulations.

The remainder of the paper is structured as follows. Section II describes the system model and interference metric. Section III touches on regulatory issues. Section IV describes a spectrum usage advertisement protocol, which can be used to optimize spectrum sharing in unlicensed and licensed bands. Section $\mathrm{V}$ gives simulation results assuming knowledge of the interference graph and selfish network behavior. Section VI concludes.

\section{SYSTEM MODEL}

Let a network $M$ be a set of terminals $X_{i}$, which implement a specific common wireless standard and want to communicate. Let $M\left\{X_{i}\right\}$ denote the network of a terminal $X_{i}$. All terminals of a given network use the same frequency interval, given by a lower cutoff frequency $f_{l}$ and a higher cutoff frequency $f_{h}$. Without loss of generality, the total available spectctrum interval is normalized to $[0,1]$. The frequency interval of a network $M_{j}$ is given by $\mathcal{F}\left\{M_{j}\right\}=\left[f_{l}, f_{h}\right] \subset$ $[0,1] . \mathcal{F}$ without argument denotes the set of all frequency assignments. 


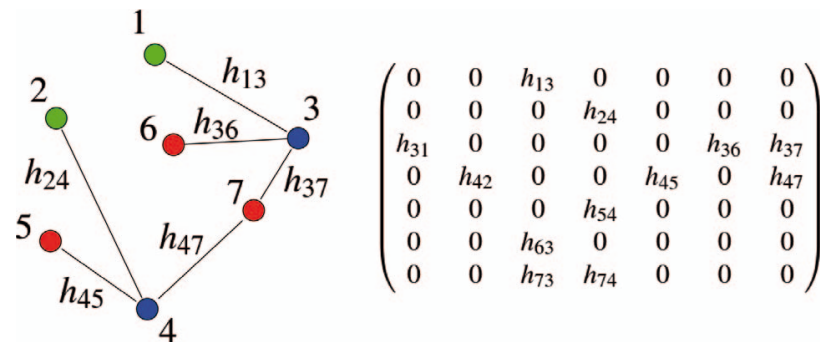

Fig. 1: Example of an interference graph and symmetric matrix for 3 networks. Red and green use orthogonal frequencies.

Between terminals $X_{i}$ and $X_{j}$ a symmetric channel with coefficients $h_{i j}=h_{j i}$ is assumed. The channel coefficients reflect the mean path loss and are written in a channel matrix $\mathbf{H}$, indexed by $i$ and $j$.

To determine which terminals cause interference to each other, one needs to know to which network a node belongs and for which networks the frequency intervals overlap. This information is given by the matrices PI and FI.

Two terminals $X_{i}$ and $X_{j}$ can interfere if they belong to different networks:

$$
(\mathbf{P I})_{i j}=\left\{\begin{array}{ll}
1 & : M\left\{X_{i}\right\} \neq M\left\{X_{j}\right\} \\
0 & : \text { otherwise }
\end{array} .\right.
$$

Two terminals $X_{i}$ and $X_{j}$ can interfere if the channels of their networks overlap in the frequency domain:

$$
(\mathbf{F I})_{i j}= \begin{cases}1 & : \mathcal{F}\left\{M\left\{X_{i}\right\}\right\} \cap \mathcal{F}\left\{M\left\{X_{j}\right\}\right\} \neq \emptyset \\ 0 & : \text { otherwise }\end{cases}
$$

This binary interference metric is motivated by the fact that any inband interference tends to have a significant impact on the receiver performance. Combining these two conditions, two terminals $X_{i}$ and $X_{j}$ interfere if and only if they belong to different networks and the frequency assignments overlap. The interference matrix $\mathbf{I}$ is then given elementwise as

$$
(\mathbf{I})_{i j}=(\mathbf{P I})_{i j}(\mathbf{F I})_{i j}(\mathbf{H})_{i j} .
$$

From the interference matrix one can generate an undirected interference graph for visualization. Figure 1 shows an example for 7 terminals and 3 networks. The optimal network resource assignment minimizes the total interference over all terminals:

$$
\mathcal{F}_{\text {opt }}=\min _{\mathcal{F}}|| \mathrm{I} \|_{\mathrm{F}}=\min _{\mathcal{F}} \sqrt{\sum_{i, j}\left|(\mathbf{I})_{i j}\right|^{2}} .
$$

where $\|\cdot\|_{F}$ denotes the Frobenius norm. To make this optimization tractable, assume a frequency discretization into $N$ channels of bandwidth $1 / N$. Then there are less ${ }^{1}$ than $N^{M}$ combinations for $M$ networks, so an optimization of (4) via exhaustive search is tractable for a small number of networks and a small number of channels. Direct optimization is of

\footnotetext{
${ }^{1}$ Note that not all center frequencies are valid at the edges of the spectrum band.
}

course not feasible as the networks do not follow a central authority and optimization is computationally expensive: a distributed algorithm has to be specified which leads to selforganization and asymptotically converges to an acceptable local minimum or even the global minimum.

\section{Model Limitations and Extensions}

The system model is simple and at the same time flexible enough to reflect realistic spectrum usage. Nevertheless, some aspects are not explicitly covered but can be included easily by extending the model. E.g., the temporal component of spectrum usage can be included by introducing a duty cycle for each terminal. The interference matrix is then weight with this duty cycle. Spectrum access by frequency hopping protocols can also be included by introducing multiple conceptual networks with low duty cycle. As mobile terminals strive to minimize their power consumption and hence transmission power, power control is another important aspect to be included in the model. This can be done in analogy to duty cycle by scaling interference with the transmit power. These extensions are not discussed further, as they do not affect the basic concept of minimizing the total interference given in (4).

\section{EXPLANATORY NOTES ON EFFICIENT USE OF SPECTRUM AND TECHNOLOGICAL NEUTRALity}

From a regulatory viewpoint, rules imposed on devices accessing spectrum should lead to efficient use of spectrum and should not favor a specific technology. In other words, regulation should be technologically neutral.

\section{Efficient Use of Spectrum}

Quantifying efficient use of spectrum is generally not trivial, as a multitude of factors, which do not necessarily need to be of technical nature, but can also include economical or political considerations, come into play. Limiting oneself to technical considerations, a practical metric to measure efficient use of spectrum can be the number of supported devices or technologies in a given bandwidth. Hence, spectrum regulation should constantly strive to create new possibilities for wireless applications, while at the same time reviewing the use of spectrum by incumbent users, safeguarding it or pushing towards technological progress when appropriate.

\section{Technological Neutrality}

To foster competition between technologies, regulation should be technologically neutral. A simple example of technological neutral regulation is the current regulation of the unlicensed ISM bands: few technological factors such as maximum transmission power or duty cycle are regulated. Any more sophisticated regulation, such as the distributed pilot channel protocol described in the next section, should also restrict itself to regulation of physical quantities. The details of the underlying protocol should not be regulated explicitly to leave room for technological development. 


\section{IMPROVED SPeCtrum Sharing VIA A Distributed COMMON PILOT CHANNEL}

The optimization problem (4) cannot be solved in a centralized manner, as this would require a central instance to decide about spectrum assignment. Hence, it has to be solved in a distributed manner and spectrum allocation needs to be implicitly, not explicitly, negotiated between networks. As terminal capabilities in terms of computational resources vary largely, excessive negotiation between terminals is also not advisable. Taking this into account, we propose to make it mandatory to advertise spectrum usage over a distributed pilot channel by a predefined protocol. Optimization of spectrum usage in the sense of (4) is then performed by each network according to its own needs and capabilities.

Assume that each terminal and each network carry a unique identifier. A node accessing spectrum regularly broadcasts a notification beacon with maximum desired transmit power on the common pilot channel and listens to the notifications of other nodes. A notification beacon includes the necessary information about spectrum usage, e.g., center frequency, occupied bandwidth and duty cycle. It is furthermore designed in such a way that accurate measurement of the channel coefficients $h_{i j}$ between nodes is possible.

Each node keeps track of the notification beacons of other nodes and broadcasts a list of all known notifications and channel coefficients with its own notification. This way, the spectrum usage information is distributed to all terminals accessing spectrum according to a flooding protocol. After a period of time, the information of all terminals accessing spectrum is available at each node in the network and an interference graph (an interference matrix I) can be built at each terminal.

A network $M_{j}$ can now infer from the interference graph, which other networks access spectrum and what the magnitude of the accumulated interference is at a given center frequency. If a network sees an advantage in changing its channel ${ }^{2}$, a new channel is chosen. A reasonable choice for the network is local greedy interference minimization over all possible network center frequencies $\mathcal{F}_{\text {net }}$ :

$$
\mathcal{F}_{\text {opt,net }}=\min _{\mathcal{F}_{\text {net }}}\|\mathrm{I}\|_{\mathrm{F}}=\min _{\mathcal{F}_{\text {net }}} \sqrt{\sum_{i, j}\left|(\mathbf{I})_{i j}\right|^{2}} .
$$

The local optimization according to (5) is a sensible and selfish choice for the network due to the fact that interference as defined in (3) is reciprocal: minimizing the interference of the network to others minimizes the interference of others to the network. This is a direct consequence of the symmetric channel.

\section{Scalability of Flooding}

The notification interval should be chosen according to the dynamics of the network and the expected user density. It should be small enough to allow for timely distribution

\footnotetext{
${ }^{2}$ Note that the concrete action a network takes to avoid interference, if any at all, should not be part of the regulation to adhere to technological neutrality.
}

of changes in the network topology, but long enough to avoid congestion of the pilot channel. With reasonable implementation assumptions and appropriately chosen notification interval, we show that such a flooding protocol is indeed stable for a high number of nodes. Assume that the pilot channel is slotted, notification beacons have fixed length ${ }^{3} T$ and each node broadcasts its notification with probability $P_{s}$ in a slot. A station entering the network now listens to the notifications of all its $n$ neighbors. The probability to receive a notification beacon of a certain neighbor during a slot is then

$$
P_{1}=P_{s}\left(1-P_{s}\right)^{n},
$$

which is exactly the probability that only one neighbor transmits. For $P_{1}$ to be maximal, choose $P_{s}=(n+1)^{-1}$. The probability to receive an arbitrary neighbor successfully is given as:

$$
n P_{1}=\left(1+\frac{1}{n}\right)^{-(n+1)} \approx \frac{1}{e} .
$$

This shows that, with the assumptions made, discovery of neighbors is sufficiently fast and the protocol is stable.

\section{Cross-layer Optimization, Hidden Nodes and Exposed Nodes}

The interference graph physical layer information cannot only be used to avoid interference by changing center frequencies, it can also be used to improve performance of higher protocol layers, taking into account the spatial interference distribution. E.g., in multi-hop networks, a routing metric can be based on the expected aggregate interference as given by the interference graph. On the medium access layer, the pilot channel effectively reduces the number of hidden nodes. By definition, a hidden node is a node not able to broadcast to any other node. It is hence not part of the interference graph and cannot cause interference and cannot be interfered with ${ }^{4}$. In a handshake protocol or carrier sense protocol, an exposed terminal is a terminal whose transmissions are blocked due to interference at the transmitter, even if there is no interference at the receiver. The pilot channel can be used to reduce the number of exposed nodes: from the interference graph, the node wanting to transmit can deduce that interference does not extend to its desired destination, if it keeps track of which node is currently using the channel.

Added Regulatory Value and Spectrum Sharing with Primary Users

From a regulatory viewpoint a distributed pilot channel is valuable as it can be used by all devices to achieve more efficient spectrum usage, avoiding spectrum sensing and detection problems. This is however not the only regulatory benefit: in bands with incumbent primary users who need protection, it is easy to implement a "quiet" beacon signal to grant or deny access in certain areas to certain frequency bands. This allows for coexistence with quality guarantees to

\footnotetext{
${ }^{3}$ This limits the maximum number of allowable hops and direct neighbors.

${ }^{4}$ This is a consequence of the precondition that the notification beacon covers an area greater than the interference area. Of course, nodes not advertising their spectrum usage are still hidden to the network.
} 


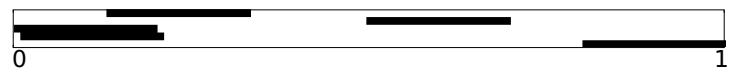

(a) Initial spectrum usage

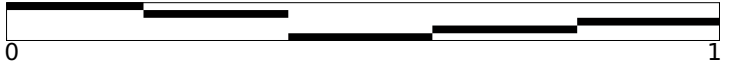

(b) After convergence

Fig. 2: Frequency orthogonalization by local greedy optimization

primary users. The operator-assisted beacon approach has been studied by Mangold et al. in [7].

Costs

Support for a distributed pilot channel requires significant effort in standardization. Devices supporting a pilot channel as described here will ideally have to support another signal path, which means extra costs in terms of signal processing. If concurrent access to spectrum and the pilot channel is needed, the high-frequency frontend will have to become more complex as well.

\section{Simulations}

Based on the assumption that a working protocol according to Section IV is implemented, we simulate typical network setups to show the improvement in spectrum sharing. Exact knowledge of the interference graph at each terminal is assumed. The channel coefficients are generated according to a physical model [8]. For nodes $X_{i}$ and $X_{j}$ the channel coefficient $h_{i j}$ is given as

$$
h_{i j}=\frac{1}{\left|\mathrm{P}\left\{X_{i}\right\}-\mathrm{P}\left\{X_{j}\right\}\right|^{\alpha}},
$$

where $\mathrm{P}\left\{X_{i}\right\}$ denotes the geometrical position of a terminal $X_{i}$ and $\alpha$ denotes the path loss exponent with $\alpha \in[2,4]$.

Each network uses a local greedy optimization strategy according to (5). If there are multiple center frequencies with equivalent interference metric to choose from, the lowest center frequency is chosen. This avoids spectrum fragmentation effects. For each network, the local optimization is easily tractable as there are less than $N$ hypotheses to test ${ }^{5}$.

\section{Minimizing Interference via Frequency Orthogonalization}

In an interference limited environment the optimal signalization strategy is known to be orthogonal signalization [3]. Regardless of the network geometry, if the available total bandwidth exceeds the bandwidth required by all networks one expects orthogonal channels for the global minimum of (4). This is indeed the case and local greedy optimization with alignment always finds the global minimum. Figure 2 shows this for $N=100$ and 5 networks with a normalized bandwidth of 0.2 each.

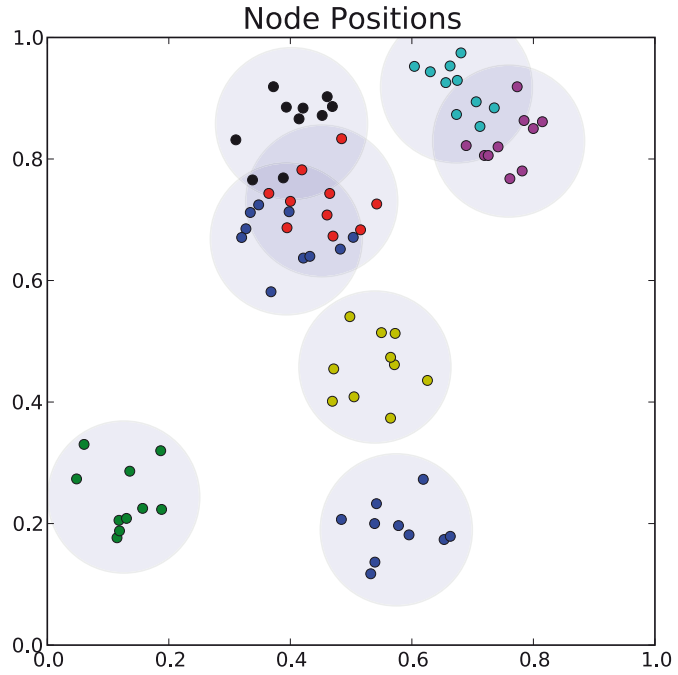

Fig. 3: Network geometry

\section{Minimizing Interference via Spatial Frequency Reuse}

If the aggregate bandwidth of all networks exceeds the available bandwidth, networks have to share spectrum. The optimal solution according to (4) then naturally promotes appropriate frequency reuse in space. Close networks will use orthogonal frequencies, whereas spatially separated networks will share common channels. Figure 3 shows the geometry of 8 networks using a bandwidth of 0.2 each. The initial randomized spectrum usage is given in Figure 4. After convergence of the optimization algorithm, the local minimum shown in Figure 5 is found. Close networks causing high interference now use orthogonal channels and these channels are aligned, minimizing interference. Comparing Figure 4 and Figure 5, the overall reduction of interference is visible by a shift towards "colder" colors.

An important question to be answered is the quality of the local minima found. As seen in Figure 6, the greedy optimization converges, given a random initial spectrum allocation, after 40 iterations and is close to optimum even in the worst case.

\section{CONCLUSION}

A distributed pilot channel based on advertisement of spectrum usage as described in this paper can be used to improve spectrum sharing in unlicensed or licensed bands. For coexistence of wireless devices using heterogeneous technologies with different priorities, it is simple to implement a "quiet" beacon to restrict access to spectrum in certain areas. From the standpoint of a regulatory body, this also avoids the cumbersome verification of the spectrum sensing or primary user detection capabilities of devices, a complicated and unsolved issue.

\footnotetext{
${ }^{5}$ Local greedy optimization requires iterative testing of just $N$ hypotheses instead of $N^{M}$
} 


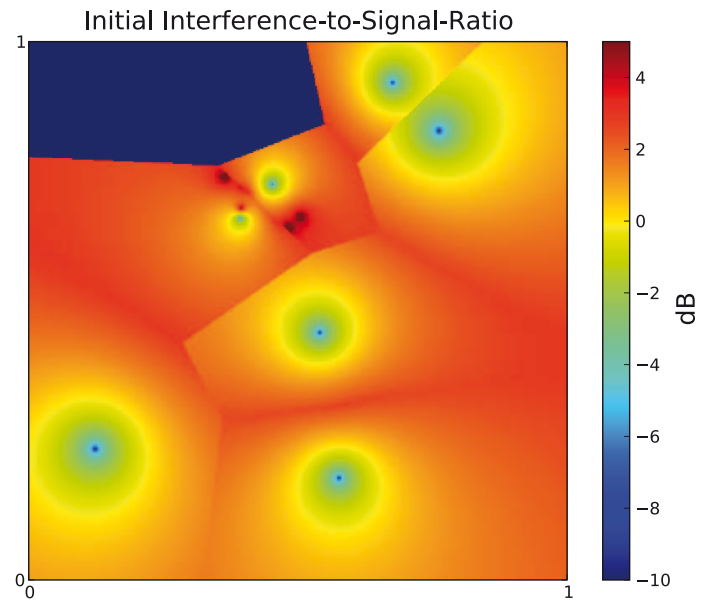

(a) Initial interference-to-signal ratio

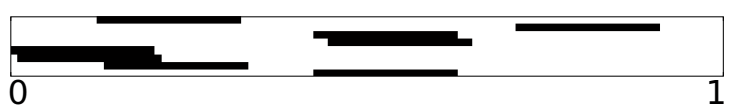

(b) Initial spectrum usage

Fig. 4: Initial spectrum usage, $\alpha=2$. Each point in the plane is assigned to the network closest to it, resulting in the spatial tesselation shown. The interference-to-signal-ratio (ISR) is plotted for all networks and given by the aggregate interference divided by the signal level of the network. This signal level is given by a (virtual) node transmitting at unit power and located at the barycenter of the network.

As the physics of wave propagation determine the playing field for spectrum sharing, a distributed pilot channel as described here has to be implemented as a physical channel close in frequency to the bands of interest to allow accurate measurements of channel coefficients. Since this is an appropriate and simple step towards more flexible spectrum usage, effort should be directed at standardizing such a technologyneutral interface.

Due to the fact that performance of wireless networks can be improved by supporting a distributed pilot channel, there is an incentive for device manufacturers to back such an approach and implement the added complexity. Potential gains lie in distributed spectrum sharing with primary systems, if backed by regulation, and optimization of spectrum resources in an interference limited environment.

\section{ACKNOWLEDGEMENT}

This work was supported by the Federal Ministry of Economics and Technology (BMWi) under project number 38/08: Effiziente Frequenznutzung im TK-Review. The views expressed are those of the authors and do not necessarily represent those of the BMWi or BMWi policy.

\section{REFERENCES}

[1] "IEEE Standards Coordinating Committee 41 (Dynamic Spectrum Access Networks)," http://www.scc41.org.

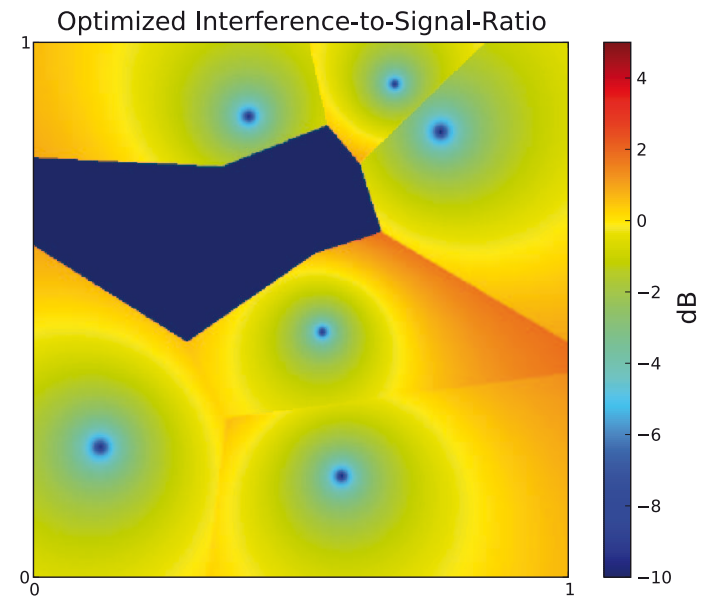

(a) Optimized interference-to-signal ratio

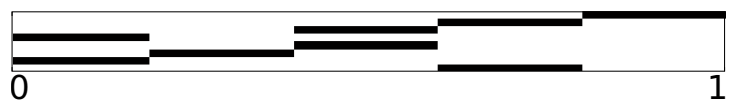

(b) Optimized spectrum usage

Fig. 5: After convergence of local greedy optimization, $\alpha=2$.

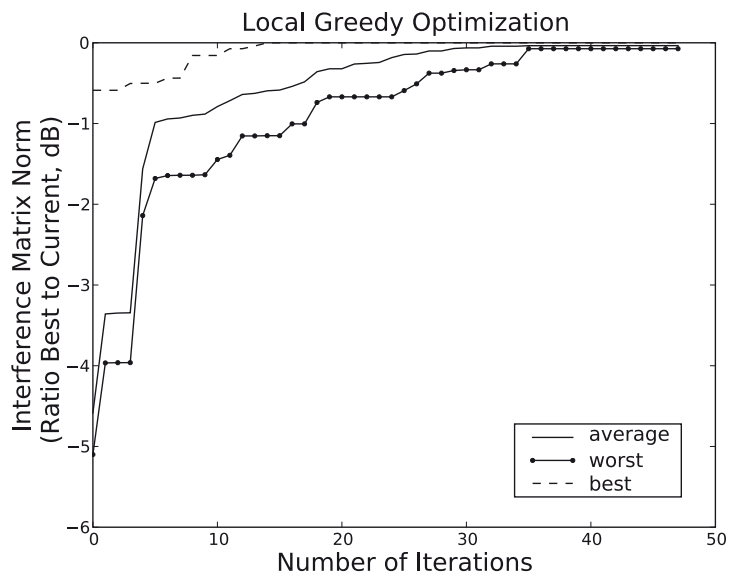

Fig. 6: Norm of the interference matrix relative to the global optimum with local greedy optimization, $\alpha=2$. Initial spectrum usage is random. An iteration is a change of center frequency of a network. Plotted are the best, worst and average performance on each iteration for 100 runs.

[2] S. Buljore, P. Houze, O. Holland, and T. Farnham, "Architecture and Enablers for Optimized Radio Resource Usage in Heterogeneous Wireless Access Networks: The IEEE 1900.4 Working Group,' IEEE Communications, Jan. 2009.

[3] R. Etkin, A. Parekh, and D. Tse, "Spectrum Sharing for Unlicensed Bands," IEEE Journal on Selected Areas in Communications, Apr. 2007.

[4] L. Grokop and D. Tse, "Spectrum sharing between wireless networks," 2008. [Online]. Available: http://www.citebase.org/abstract?id=oai:arXiv.org:0809.2840

[5] J. Zhao, H. Zheng, and G.-H. Yang, "Distributed coordination in dynamic spectrum allocation networks," First IEEE International Symposium on New Frontiers in Dynamic Spectrum Access Networks (DySPAN), Nov. 2005. 
PROCEEDINGS OF THE 4th INTERNATIONAL CONFERENCE ON CROWNCOM 2009

[6] C. Peng, H. Zheng, and B. Y. Zhao, "Utilization and fairness in spectrum assignment for opportunistic spectrum access," Mob. Netw. Appl., vol. 11, no. 4, 2006.

[7] S. Mangold, A. Jarosch, and C. Monney, "Operator assisted cognitive radio and dynamic spectrum assignment with dual beacons - detailed evaluation," First International Conference on Communication System Software and Middleware, 2006.

[8] P. Gupta and P. Kumar, "The capacity of wireless networks," IEEE Transactions on Information Theory, Mar 2000. 